\title{
Application of norm-conserving pseudopotentials to intense laser-matter interactions
}

\author{
Xiao-Min Tong, ${ }^{1,2, *}$ Georg Wachter, ${ }^{3}$ Shunsuke A. Sato, ${ }^{2}$ Christoph Lemell, ${ }^{3}$ Kazuhiro Yabana, ${ }^{1,2}$ and Joachim Burgdörfer ${ }^{3}$ \\ ${ }^{1}$ Center for Computational Sciences, University of Tsukuba, Tsukuba 305-8577, Japan \\ ${ }^{2}$ Graduate School of Pure and Applied Sciences, University of Tsukuba, Tsukuba 305-8571, Japan \\ ${ }^{3}$ Institute for Theoretical Physics, Vienna University of Technology, 1040 Vienna, Austria
}

(Received 20 July 2015; published 29 October 2015)

\begin{abstract}
We investigate the applicability of norm-conserving pseudopotentials to intense laser-matter interactions by performing time-dependent density functional theory simulations with an all-electron potential and with norm-conserving pseudopotentials. We find pseudopotentials to be reliable for the simulation of above-threshold ionization over a broad range of laser intensities both for the total ionization probability and the photoelectron energy spectrum. For the simulation of high-order-harmonic generation, pseudopotentials are shown to be applicable for lower-order harmonics in the spectral range in which the one-photon recombination dipole-matrix element can be recovered by the pseudopotential calculation.
\end{abstract}

DOI: 10.1103/PhysRevA.92.043422

PACS number(s): 32.80.Rm, 31.15.ee, 71.15.Dx

\section{INTRODUCTION}

Intense laser-matter interaction is one of the current hot topics in physics due to the rapid advances of laser technology [1-4]. In principle, the dynamics can be studied by solving the many-electron time-dependent Schrödinger equation. However, apart from a few simple systems like hydrogen or helium it is still very challenging to perform a full quantum simulation for many-electron systems. Therefore many approximate theoretical models ranging from classical and semiclassical to perturbative methods have been proposed to interpret experimental observations or to predict physical processes in laser-matter interactions. The difficulties of $a b$ initio simulations result from the numerical efforts and the amount of computational resources needed that are far beyond the computational power available even for today's supercomputers. Density functional theory (DFT) [5-7] and time-dependent density functional theory (TDDFT) [8-11] often provide a useful compromise between efficiency and reliability for studying the electronic structures and dynamical processes of many-electron systems. However, even with TDDFT, all-electron (AE) simulations for atoms, molecules, and solids in an intense laser field are still very challenging $[10,12-15]$ because of the extensive computational resources needed.

For moderately strong laser fields [e.g., with intensities $I \leqslant 10^{15} \mathrm{~W} / \mathrm{cm}^{2}$ for a near-infrared (NIR) wavelength of $\lambda=800 \mathrm{~nm}]$ only the valence and subvalence shell electrons effectively contribute to the strong-field response of matter to the light field. Therefore, representing within a TDDFT simulation the core electrons by pseudopotentials rather than performing an all-electron calculation promises to considerably reduce the numerical effort required. Moreover, it bypasses the need to represent the fast oscillations of valence orbitals in the core region. The latter poses an obstacle to efficiently discretizing the configuration space by equispaced grids. For laser-atom interactions, the generalized pseudospectral method [16] employs nonequidistant grid distributions allowing thereby the representation of rapidly varying wave

\footnotetext{
*tong.xiaomin.ga@u.tsukuba.ac.jp
}

functions near the nucleus. This method reaches its limit for strong long-wavelength laser fields because a large number of grid points are needed. While it has been meanwhile applied to diatomic molecules by using spheroidal coordinates $[13,17,18]$ it cannot be easily extended to general many-atom systems. One characteristic feature of many-atom systems in an intense laser field is that many continuum states are involved and the ejected electron may propagate far away from its parent core before returning at a later time. Thus the conventional quantum-chemistry codes, such as GAMESS [19] or BIGDFT [20], while frequently employed in structure and spectral calculations, cannot be easily applied to intense laser-matter interactions. Treating such problems with $\mathrm{AE}$ methods becomes increasingly impractical since too many grid points are needed to describe the fast oscillation of the wave function in the inner region, and the wave function extends to very large space. For extended condensed-matter systems with periodic-boundary conditions, the use of equispaced grids in both coordinate and $k$ space and of pseudopotentials is key to effectively describing strong-field laser-matter interactions.

Although pseudopotentials are frequently used for electronic structure simulations in large molecules, clusters, and condensed matter and for dynamical processes in the perturbative limit [21,22], the validity of the pseudopotentials in intense laser-material interactions beyond the perturbative regime has not yet been tested systematically. The purpose of the present paper is to explore the applicability of pseudopotentials to describing two prominent signatures of strong-field response of matter: above-threshold ionization (ATI) and high-orderharmonic generation (HHG). As a test case for an atomic many-electron system we consider in the following argon for which we perform comparative all-electron and pseudopotential (PP) calculations within the framework of TDDFT. In our study we employ norm-conserving pseudopotentials (NCPP) [23-26] which feature the distinct advantage of yielding the same Kohn-Sham (KS) energy levels and the same scattering phase (modulo $\pi$ ) for low-energy electron scattering as the ones from AE simulations. NCPPs can be generated from all-electron DFT simulations [27] and have been applied in the past to a variety of dynamical processes for many-electron systems from atoms [28], molecules [22], and clusters [29] to condensed matter [30]. Justification of NCPP to many-electron 
dynamics in the perturbative regime has been investigated by Yabana and Bertsch [31].

By comparing the strong-field response treated by TDDFTAE and TDDFT-PP, we demonstrate that NCPPs work well for above-threshold ionization over a broad range of laser intensities. For high-order-harmonic generation, NCPPs work well for lower harmonics with energies $E$ below a critical value above which the photorecombination matrix element into pseudostates deviates from that for recombination into AE orbitals. Implications for the simulation of ATI and HHG spectra in many-electron systems will be discussed.

An outline of the methods employed will be given in Sec. II followed by the presentation of the application to ATI and HHG in argon in Sec. III. Atomic units are used unless specified otherwise.

\section{THEORETICAL METHODS}

Laser-matter interactions can be simulated within the framework of TDDFT by solving the time-dependent KohnSham (TDKS) equations

$$
i \frac{\partial \psi_{i}(\mathbf{r}, t)}{\partial t}=H(\mathbf{r}, t) \psi_{i}(\mathbf{r}, t)
$$

with $\psi_{i}(\mathbf{r}, t)$ the time-dependent $i$ th orbital wave function $(i=1, \ldots, N)$ and $H(\mathbf{r}, t)$ the single-particle-like Kohn-Sham [6] Hamiltonian. The time-dependent electron density is expressed as

$$
\rho(\mathbf{r}, t)=\sum_{i=1}^{N} n_{i} \psi_{i}^{*}(\mathbf{r}, t) \psi_{i}(\mathbf{r}, t),
$$

with $n_{i}$ the electron occupation number of the $i$ th orbital and $N$ the number of orbitals. For an all-electron calculation

$$
\sum_{i=1}^{N} n_{i}=N_{\mathrm{e}}
$$

with $N_{\mathrm{e}}$ the total number of electrons $\left(N_{\mathrm{e}}=18\right.$ in the present case). For atoms, the Hamiltonian can be written as

$$
H(\mathbf{r}, t)=-\frac{1}{2} \nabla^{2}+V_{\mathrm{eff}}[\rho(\mathbf{r}, t)]+V_{\mathrm{ext}}(\mathbf{r}, t),
$$

where $V_{\text {ext }}(\mathbf{r}, t)$ is the electron-laser interaction and $V_{\text {eff }}[\rho(\mathbf{r}, t)]$, the effective potential,

$$
V_{\mathrm{eff}}[\rho(\mathbf{r}, t)]=-\frac{Z}{r}+\int \frac{\rho\left(\mathbf{r}^{\prime}, t\right)}{\left|\mathbf{r}-\mathbf{r}^{\prime}\right|} d \mathbf{r}^{\prime}+V_{\mathrm{xc}}[\rho(\mathbf{r}, t)],
$$

with $Z$ the charge of the nucleus. The first term of the above equation stands for the electron-nuclear Coulomb interaction, the second term is the direct (or Hartree) electron-electron interaction, and the third term represents the exchange-correlation interaction which is a universal functional of the electron density the exact form of which is still unknown. A wide variety of approximate forms for $V_{\mathrm{xc}}$ have been proposed. Since the focus of the present paper is not to identify optimal choices for argon, we choose in the following the simplest and most widely used local density functional [32,33], which has been shown to predict most of the physical processes at least qualitatively correctly. For comparing with experiments quantitatively, specifically tailored exchange-correlation functionals, such as the meta-GGA functional [34,35], could be used.

We propagate the time-dependent wave functions by using the second-order split-operator method in the energy representation [36] as

$$
\psi_{i}(\mathbf{r}, t+\Delta t)=e^{-i H_{0} \Delta t / 2} e^{-i V(\mathbf{r}, t) \Delta t} e^{-i H_{0} \Delta t / 2} \psi_{i}(\mathbf{r}, t),
$$

where $H_{0}$ is the time-independent laser field free atomic Hamiltonian which in the present case is taken as

$$
H_{0}=-\frac{1}{2} \nabla^{2}+V_{\text {eff }}\left[\rho_{0}(\mathbf{r})\right],
$$

with $\rho_{0}(\mathbf{r})$ the ground-state atomic electron density in the absence of the external laser field. Consequently, the interaction potential $V(\mathbf{r}, t)$ in Eq. (6) contains, in addition to the interaction with the external field, $V_{\text {ext }}$, also the interaction with the time-dependent charge density fluctuation $\delta \rho(\mathbf{r}, t)$ of the many-electron system induced by the external field,

$$
\begin{aligned}
V(\mathbf{r}, t) & =V_{\mathrm{ext}}(\mathbf{r}, t)+V[\delta \rho(\mathbf{r}, t)] \\
& =V_{\mathrm{ext}}(\mathbf{r}, t)+\int \frac{\delta \rho\left(\mathbf{r}^{\prime}, t\right)}{\left|\mathbf{r}-\mathbf{r}^{\prime}\right|} d \mathbf{r}^{\prime}+\delta V_{\mathrm{xc}}(\mathbf{r}, t),
\end{aligned}
$$

with $\delta \rho(\mathbf{r}, t)=\rho(\mathbf{r}, t)-\rho_{0}(\mathbf{r}, t)$ and $\delta V_{\mathrm{xc}}(\mathbf{r}, t)=V_{\mathrm{xc}}[\rho(\mathbf{r}, t)]-$ $V_{\mathrm{xc}}\left[\rho_{0}(\mathbf{r})\right]$, the effective exchange-correlation potential due to the density variation relative to the initial density. We refer to $V[\delta \rho(\mathbf{r}, t)]$ in the following as the dynamical screening potential. In the all-electron TDDFT we calculate $V_{\text {eff }}\left[\rho_{0}(\mathbf{r})\right]$ for all electrons [Eq. (5)] self-consistently without the laser field. Accordingly, the dynamical screening potential $V[\delta \rho]$ accounts for the response and density fluctuations of all electrons in the system.

For the calculation employing NCPPs we represent the electrons in inner shells $(n=1,2)$ in terms of pseudopotentials. In turn, the dynamical screening potential $V^{\mathrm{PP}}[\delta \rho]$ describes the response of the $3 s$ and $3 p$ electrons only. Accordingly, the all-electron ground-state potential $V_{\text {eff }}\left[\rho_{0}\right]$ in Eq. (7) is replaced by nonlocal $\ell$-dependent pseudopotentials $V_{\ell}^{\mathrm{PP}}$,

$$
V_{\mathrm{eff}}^{\mathrm{PP}}(\mathbf{r})=\sum_{\ell, m} V_{\ell}^{\mathrm{PP}}(r)\left|Y_{\ell}^{m}\right\rangle\left\langle Y_{\ell}^{m}\right| \text {. }
$$

The pseudopotential for each $\ell$ sector is calculated from the inversion of the Schrödinger equation for the corresponding radial pseudo-wave-function $R_{\ell}^{\mathrm{PP}}(r)$,

$$
V_{\ell}^{\mathrm{PP}}(r)=\varepsilon_{n \ell}-\frac{\ell(\ell+1)}{2 r^{2}}-\frac{1}{2 r R_{\ell}^{\mathrm{PP}}(r)} \frac{d^{2}}{d r^{2}}\left[r R_{\ell}^{\mathrm{PP}}(r)\right],
$$

where $\varepsilon_{n \ell}$ is the Kohn-Sham energy eigenvalue of the $n=3$ electrons from the AE calculation. The pseudo-wave-functions $R_{\ell}^{\mathrm{PP}}(r)$ are constructed subject to the constraints that outside a cutoff radius $r_{\text {cut }}\left(r_{\text {cut }}=2\right.$ a.u. in the calculations), the $R_{\ell}^{\mathrm{PP}}(r)$ are identical to the corresponding AE Kohn-Sham orbitals $R_{\ell}(r)$. Within the $r_{\text {cut }}$ sphere the probability (or charge) of the AE orbital and the PP orbital must agree,

$$
\int_{0}^{r_{\mathrm{cut}}} d r\left|R_{\ell}^{\mathrm{PP}}(r)\right|^{2} r^{2}=\int_{0}^{r_{\mathrm{cut}}} d r\left|R_{\ell}(r)\right|^{2} r^{2},
$$

in order to conserve the norm of the wave function. We construct a smooth nonoscillating pseudo-wave-function for a given $\ell$ as $r^{\ell} \exp [p(r)]$ with $p(r)$ a polynomial. Matching both the pseudo-wave-function and its derivative to the $\mathrm{AE}$ wave 


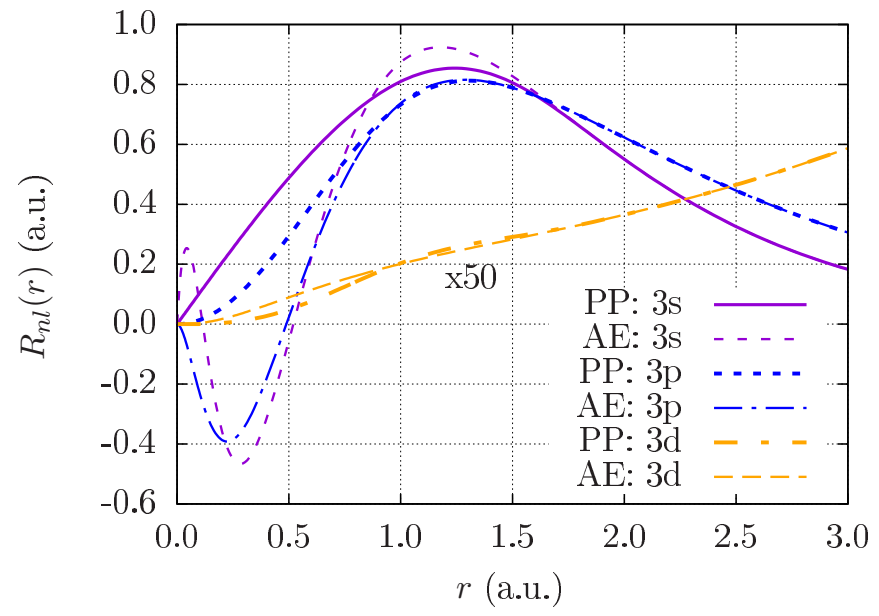

FIG. 1. (Color online) Radial (pseudo-)wave functions of Ar3s, $3 p$, and $3 d$ orbitals calculated by DFT with the all-electron potential and norm-conserving pseudopotentials. The $3 d$ wave functions are multiplied by 50 .

function at $r=r_{\text {cut }}$ determines the pseudo-wave-functions (Fig. 1) and, via Eq. (10), the pseudopotentials (Fig. 2).

In our simulation we explicitly calculate $V_{\ell}^{\mathrm{PP}}(r)$ for the first three partial waves $(\ell=0,1,2)$ and assume that higher $\ell$ bound states or partial waves in the continuum excited by the external driving field $V_{\text {ext }}$ are well represented by the $d$-wave channel potential since core penetration is strongly suppressed (see Fig. 1). Upon replacing $V_{\text {eff }}\left[\rho_{0}\right]$ in Eq. (7) by $V_{\text {eff }}^{\mathrm{PP}}(\mathbf{r})$ [Eq. (9)] and $V[\delta \rho]$ by the corresponding dynamical response potential for the pseudodensity, $V^{\mathrm{PP}}[\delta \rho]$, the propagation [Eq. (6)] proceeds completely analogously to the AE simulation.

We will also compare the TDDFT simulations with the solution of the time-dependent Schrödinger equation in the single-active electron approximation (TDSAE). In this limit, the dynamical response of the electrons is completely neglected, $V[\delta \rho]=0$, and Eq. (8) reduces to

$$
V(\mathbf{r}, t)=V_{\mathrm{ext}}(\mathbf{r}, t) .
$$

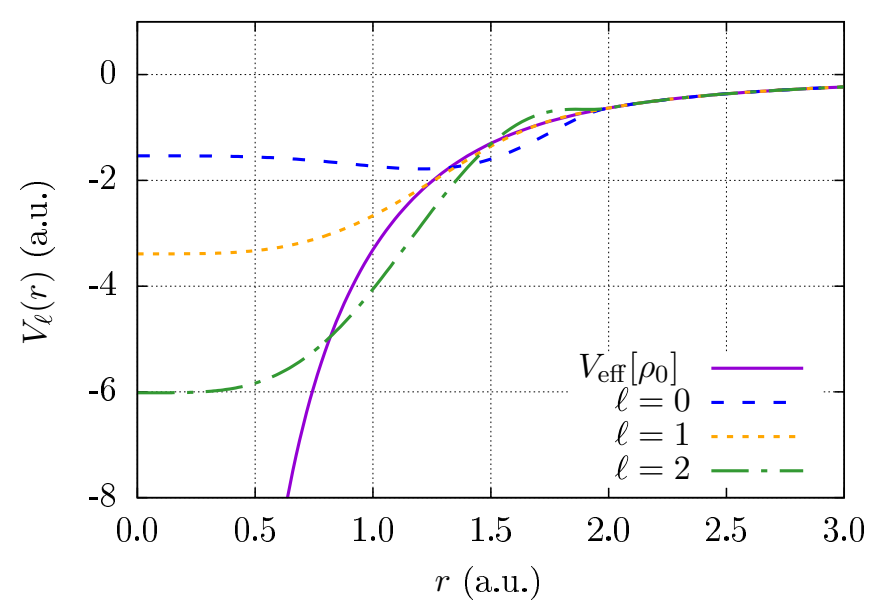

FIG. 2. (Color online) All-electron effective potential $V_{\text {eff }}\left[\rho_{0}\right]$ and norm-conserving pseudopotentials $V_{\ell}^{\mathrm{PP}}(r)$ from DFT simulations for Ar atoms.
The single active electron is propagated in the effective potential [Eq. (9)]. Therefore, by changing the effective input potential and by turning on or off the dynamical screening potential we can study laser-induced dynamical processes within the framework of either the TDSAE or the TDDFT, the latter on both the AE and NCPP levels using the same computer code.

\section{APPLICATION TO STRONG-FIELD LASER-ATOM INTERACTIONS}

\section{A. Above-threshold ionization}

One prominent signature of the highly nonlinear electronic response to strong laser-matter interaction is above-threshold ionization. In our ATI simulation we project, after each time step of propagation, the wave functions $\psi_{i}(\mathbf{r}, t)$ in the outer region $\left(r>R_{V}\right)$ onto Volkov states [37-39] in momentum space which allows one to propagate analytically the wave-packet component residing in the outer region until the conclusion of the laser pulse. After the termination of the laser pulse the inner and outer components of the wave packet are projected onto the atomic continuum states $\left\{\phi_{\ell}(\epsilon, r)\right\}$, which are normalized on the energy scale. The atomic continuum wave functions $\left\{\phi_{\ell}(\epsilon, r)\right\}$ at the energy $\epsilon$ are numerically determined for the given AE potential or NCPPs. The projection coefficients contain all information on the ATI electron spectrum as detailed in Refs. [40-42].

In the simulation, we use 80 partial waves, 2000 radial grid points, and a simulation-box size of $R_{\max }=240$ a.u., which is about 10 times the electron quiver distance $E_{0} / \omega^{2}$ for the highest intensity in the present work. Here $E_{0}$ is the peak laser electric field and $\omega$ the laser center wavelength. The quiver radius is the amplitude of a classical free electron oscillating in the laser field. In the following we present numerical results for a prototypical set of parameters for the laser field with pulse duration of $\tau=6$ fs (FWHM intensity) and central wavelength $\lambda=800 \mathrm{~nm}$. We have checked that the PP method is applicable over a wide range of wavelengths (between 400 and $1600 \mathrm{~nm}$ ). The border between the inner and outer Volkov region is set at $R_{V}=2 / 3 R_{\max }$, i.e., about the sixfold quiver radius to ensure that laser-driven recollision with the ionic core is properly represented.

The substate-resolved ionization probabilities (Fig. 3) of electrons initially occupying the $3 s, 3 p_{0}$, and $3 p_{1}$ orbitals display near-perfect agreement between $\mathrm{AE}$ and PP simulations. The inner-shell ( $1 s, 2 s, 2 p$ of Ar atoms) contributions can be completely neglected due to large ionization potentials. The total ionization probability is therefore simply the sum of all subvalence contributions.

While the identification of individual Kohn-Sham orbitals with electronic states is arguable, the KS orbitals provide a useful starting point for the qualitative interpretation of dynamical processes in atoms and molecules [43]. Irrespective of this ambiguity the total ionization probability is a welldefined observable within TDDFT. The discrepancies between the TDDFT-AE and TDDFT-PP are less than a few percent. The largest discrepancy appears for the $3 s$ valence shell at the lowest intensity for which the ionization probability is 


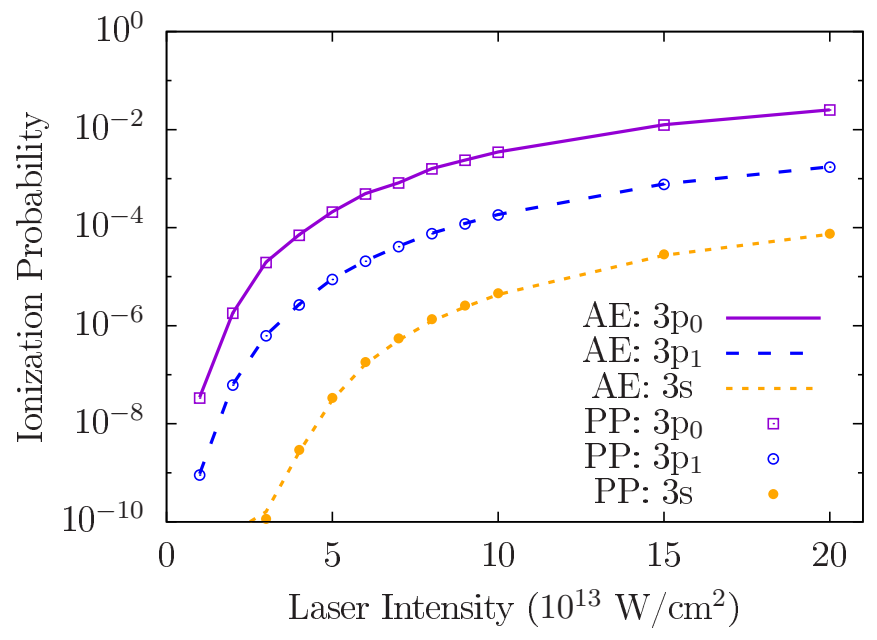

FIG. 3. (Color online) Substate resolved ionization probabilities in Ar for electrons initially occupying the $3 p_{0}, 3 p_{1}$, and $3 s$ orbitals in intense laser fields calculated by TDDFT with the all-electron potential and norm-conserving pseudopotentials. Laser parameters: $\lambda=800 \mathrm{~nm}$, pulse duration $\tau=6 \mathrm{fs}$.

exceedingly small (less than $10^{-10}$ ). This discrepancy may reflect, in fact, numerical errors.

Figure 3 also shows that ionization is dominated by the $3 p_{0}$ contribution followed by $3 p_{1}$ while the $3 s$ orbital contributes little. Although the $3 p_{0}$ and $3 p_{1}$ orbitals have the same ionization potential, the electron aligned along the laser field can be most easily ionized. The difference between $3 p$ and $3 s$ are due to differences in the ionization potentials. The larger ionization potential results in a smaller ionization probability. These findings agree with those previously reported [44-46].

Solving the time-dependent Schrödinger equation with an SAE model potential is frequently used to investigate the dynamical processes in laser-atom interactions because of its simplicity. We therefore compare for argon the ionization probabilities from a TDDFT and from a TDSAE calculation (Fig. 4). We find that the ionization probabilities of $\operatorname{Ar} 3 p_{0}$ and

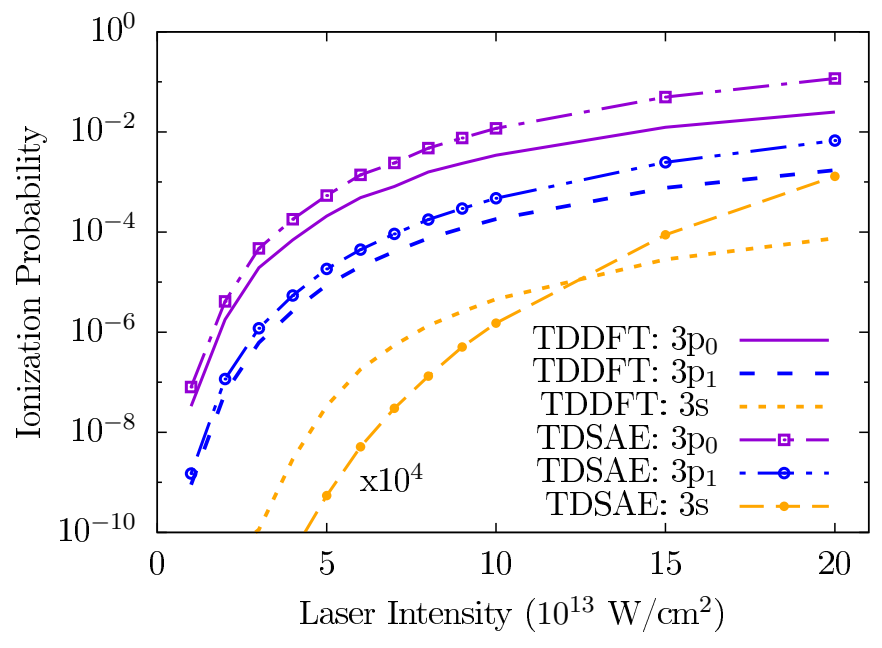

FIG. 4. (Color online) As in Fig. 3, but comparing the TDDFT$\mathrm{AE}$ and TDSAE calculations.

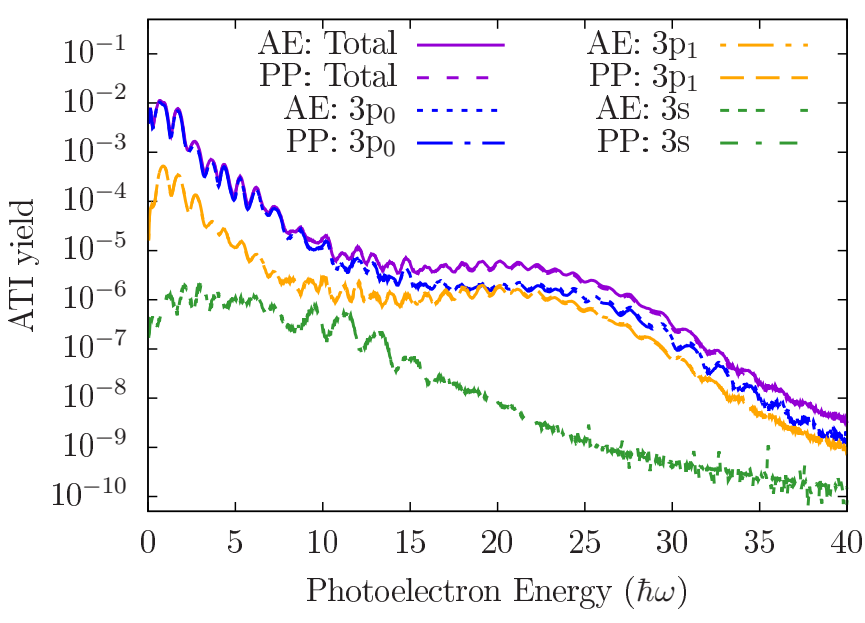

FIG. 5. (Color online) ATI spectra of Ar in an intense laser pulse calculated by TDDFT with the all-electron potential and norm-conserving pseudopotentials. Laser parameters: $\lambda=800 \mathrm{~nm}$, $I=7 \times 10^{13} \mathrm{~W} / \mathrm{cm}^{2}$, pulse duration $\tau=6$ fs.

Ar $3 p_{1}$ from TDDFT and the TDSAE model are close to each other at low intensities $\left(I \sim 10^{13} \mathrm{~W} / \mathrm{cm}^{2}\right)$ but rapidly diverge from each other at higher intensities. These discrepancies can be attributed to the dynamical screening of the laser field included in TDDFT [Eq. (8)] but absent in the TDSAE model. More specifically, the dynamic screening due to the displacement of the electron density, or polarization induced by the external laser field, reduces the ionization probability by a factor of 2 to 5 at low intensities as reported in Ref. [22]. For higher intensities when ionization probability is already significant, the suppression of the ionization in TDDFT can be attributed to the increase of the ionization potential due to the partial ionization [10]. Even though the fractional ionization is a conceptual problem of TDDFT, the reasonable agreement between simulations [10,47] and measurements $[48,49]$ indicates that dynamical screening is reasonably well accounted for by TDDFT on a mean-field level.

For the ionization probability of $\mathrm{Ar} 3 s$, the two results differ by orders of magnitude. In the TDSAE simulation, the ionization probability from $\mathrm{Ar} 3 s$ increases rapidly with intensity, but still remains much smaller than the one from TDDFT. The larger ionization probability in TDDFT can be attributed to indirect field ionization or an "antiscreening" effect due to the induced charge-density fluctuations in the valence shell. This can be viewed as the signature of electron rescattering on the mean-field level. It is also the precursor of nonsequential multiple ionization observed in experiment [50-52] and attributed to electron rescattering [53-55]. The nonsequential multiple ionization has been studied by TDDFT [12].

Photoelectron spectra (Fig. 5) can provide more detailed information on the ionization process. Obviously, the PP calculation can reproduce the spectrum determined by the AE calculation very well. In fact, the TDDFT-PP calculations yield smoother and less noisy spectra than the TDDFT-AE simulation which is a direct consequence of the much smaller number of grid points needed in the PP calculation to achieve converged results. This observation underscores the advantage 
in performing TDDFT-PP calculations for many-electron systems. At this moderate laser intensity the spectrum features the characteristic ATI peaks with $\hbar \omega$ spacing, the first cutoff for direct electrons at $2 U_{p}\left(U_{p}=E_{0}^{2} / 4 \omega^{2}\right.$, the ponderomotive energy) and the cutoff for rescattering electrons at $10 U_{p}$ [56]. At higher intensities the ATI peaks get blurred (not shown) but the TDDFT-PP method works equally well.

Although the wave functions and the effective potentials of the AE and PP calculations differ significantly as shown in Figs. 1 and 2, the resulting ionization probabilities and the ATI spectra are in good agreement with each other for a broad range of laser intensities. This can be understood in terms of a semiclassical three-step model applied to ATI: tunneling followed by electron acceleration in the laser field [57] and, for high-energy electrons, laser-driven rescattering at the ionic core. The tunneling ionization depends on the potential and the electron density near the tunneling barrier which is in the outer region $r>r_{\text {cut }}$ where the AE and NCPP potentials coincide. Also the laser field accelerates the electron in the outer region in which the potentials are the same. Finally, for the rescattering process, the PP potentials are constructed such that the scattering phase of the full AE potential is reproduced (modulo $\pi$ ) for low-energy scattering. All of this explains that the rapid oscillations of the initial wave function in the core region are of little importance for the strong-field ionization process.

\section{B. High-order-harmonic generation}

High-order-harmonic generation is the second prominent signature of nonlinear laser-matter interactions, and it is the process underlying the new ultrafast extreme ultraviolet light source [58-60] which allows one to probe ultrafast dynamics of electrons in materials [61-63]. With the time-dependent density $\rho(\mathbf{r}, t)$, we calculate the induced dipole in length form as

$$
\mathbf{d}(t)=\int \mathbf{r} \rho(\mathbf{r}, t) d \mathbf{r},
$$

and from Eq. (13) the HHG spectrum by the Fourier transform

$$
P(n \omega)=\left|\frac{1}{\tau} \int_{-\infty}^{\infty} \mathbf{d}(t) e^{i n \omega t} d t\right|^{2},
$$

with $\tau$ the pulse duration and $n$ the harmonic order. Unlike the ATI spectra we cannot decompose the HHG spectra into contributions from different orbitals since the HHG represents a coherent sum over different orbitals as shown by Eq. (14). Therefore, we focus on the total HHG spectrum. We can also calculate the induced dipole in velocity gauge $(\mathbf{v}=$ $i[H, \mathbf{r}])$ and acceleration gauge $(\mathbf{a}=i[H, \mathbf{v}])$ as described in Refs. $[36,64,65]$. If the propagation of the wave functions is converged, the HHG spectra from the three gauges should agree with each other. Thus, comparing the HHG spectra from different gauges provides a test of the numerical accuracy of the simulation.

Since the purpose of the present investigation is to probe the validity region of a NCPP potential in HHG simulations, we focus on the single-atom response and do not consider propagation and phase-matching effects [66] that are important when comparing with experiment. The convergence of the

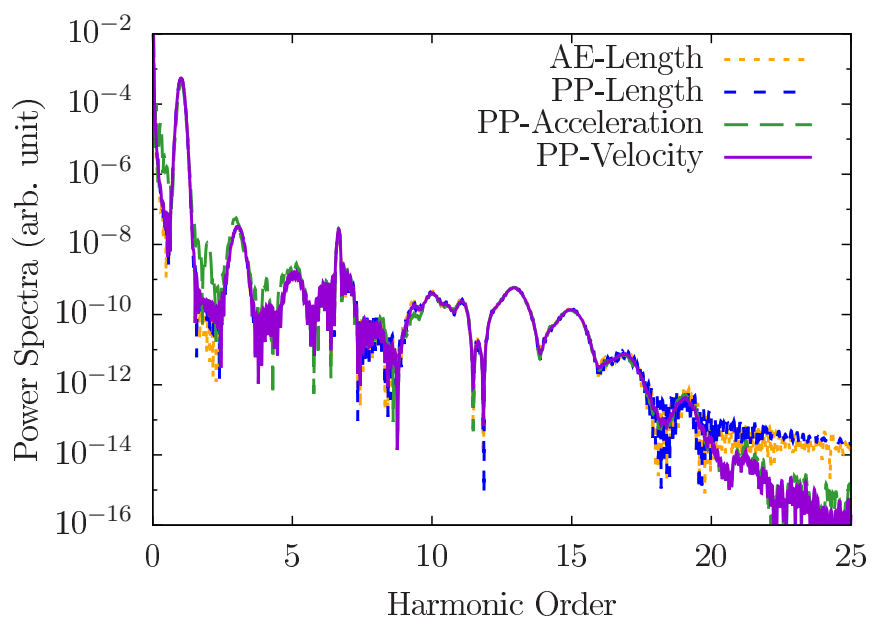

FIG. 6. (Color online) High-order harmonic generation of $\mathrm{Ar}$ atoms in an intense laser pulse calculated by TDDFT with the all-electron potential and norm-conserving pseudopotentials in length gauge. For the latter also velocity and acceleration gauge are shown. Laser parameters: $\lambda=800 \mathrm{~nm}, I=5 \times 10^{13} \mathrm{~W} / \mathrm{cm}^{2}$, pulse duration $\tau=6$ fs.

HHG spectra calculated within the full TDDFT-AE simulation in either the velocity or acceleration gauge as a function of grid-point density is slow because in these gauges the major contributions to the dipole-matrix elements come from the derivations of rapidly oscillating wave functions in the core region. By contrast, the length gauge places more weight on the outer region yielding rapid convergence as the grid density is increased. Therefore, when comparing with a PP calculation whose key advantage is low grid density we will use the length gauge for the AE calculation (Fig. 6).

Typical HHG spectra feature a rapid decrease for low-order HHG followed by a plateau terminated by a sharp cutoff consistent with the prediction by the semiclassical model $[67,68]$ for the cutoff energy $E_{\text {cut }}=I_{p}+3.17 U_{p}$, with $I_{p}$ the ionization potential. The sharp peak around $n=6.7$ is not a HHG feature but signifies the resonant transition from $4 s \rightarrow$ $3 p$. Overall, the AE spectrum agrees with the PP spectrum quite well for energies $E<n \hbar \omega$ with $n \approx 20$. Moreover, the PP simulations in length, velocity, and acceleration gauges agree with each other within this spectral range which is a consequence of the fact that the fast oscillations of the initial wave functions in the inner region are removed in NCPP calculations, pointing to the numerical advantages of pseudopotentials. Similar to previous work [36,64], we find the HHG in the velocity gauge has less background above $E_{\text {cut }}$. Therefore, in the following discussion, we present the HHG in the velocity gauge for NCPPs and in the length gauge for the AE potential.

Comparison between the TDSAE model and the TDDFT employing the same AE potential [see Eq. (7)] displays discrepancies for HHG (Fig. 7). Interestingly, the HHG spectra of TDSAE are higher than the ones of TDDFT for lower order harmonics $(n<20)$ while the opposite is true for $n>20$. This reversal can be understood with the help of the three-step model for HHG [68]: tunnel ionization, acceleration of the liberated electron, and, as a third and final step, 


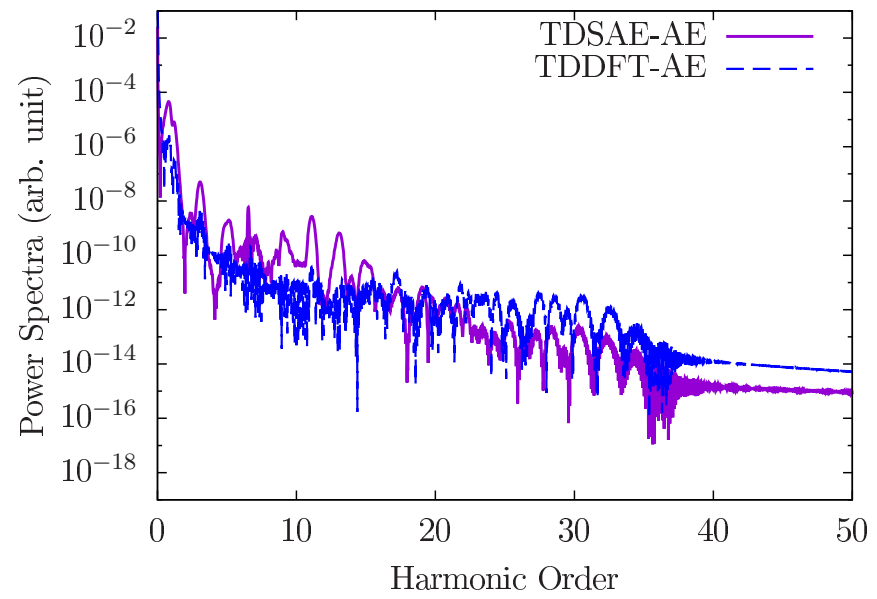

FIG. 7. (Color online) High-order-harmonic generation of $\mathrm{Ar}$ atoms calculated by TDSAE and TTDFT with all-electron potential. Laser parameters: $\lambda=800 \mathrm{~nm}, I=2 \times 10^{14} \mathrm{~W} / \mathrm{cm}^{2}, \tau=6 \mathrm{fs}$.

photorecombination with the parent ion. Within the so-called quantitative rescattering theory [69] the last step is given by the radiative recombination probability which is related to the photoionization cross section via time-reversal symmetry. The photoionization cross section in the independent-particle approximation [70] corresponding to the TDSAE model is found to be higher than the self-consistent field (SCF) cross section (corresponding to TDDFT) for lower energies $(<20 \mathrm{eV})$ while for higher photon energies $(>20 \mathrm{eV})$ the SCF cross section is larger. This general pattern is consistent with the HHG power spectra calculated with TDDFT and TDSAE although the crossover position shifts by about $10 \mathrm{eV}$, which is close to the ac-Stark shift of the ground state in the laser field.

Since the HHG originates from the radiative recombination of the electron with the parent ion it probes the overlap of the continuum wave function of the energetic electron with the initial state in the core region [68,71]. The excellent performance of TDDFT with pseudopotentials over a wide range of photoenergies is, at first glance, surprising since the pseudo-wave-function entering the recombination matrix bears little resemblance to the initial state. In order to explore the apparent insensitivity of HHG to oscillations of valence orbitals in the core region we introduce a window function

$$
w(r)= \begin{cases}0 & \text { for } \quad r<R_{c}, \\ 1-e^{-\left(r-R_{c}\right)^{2} / 25} & \text { for } r \geqslant R_{c},\end{cases}
$$

and calculate the induced dipole as

$$
\mathbf{d}(t)=\int \mathbf{r} \rho(\mathbf{r}, t) w(r) d \mathbf{r} .
$$

By varying $R_{c}$ we assess the contribution from the core region to the harmonic spectrum. Figure 8 shows the variation of the high-energy spectrum $(n>20)$ for different values of $R_{c}$. The low-order $(n<20)$ harmonic spectrum is not significantly affected at all. Up to energies in the vicinity of the cutoff energy corresponding to the $n \approx 35$ harmonic the removal of contributions from $r \leqslant R_{c}=1.5$ a.u. to the integral alters the spectrum only slightly. Dramatic variations appear only at the very-high-energy end of the spectrum beyond the cutoff.

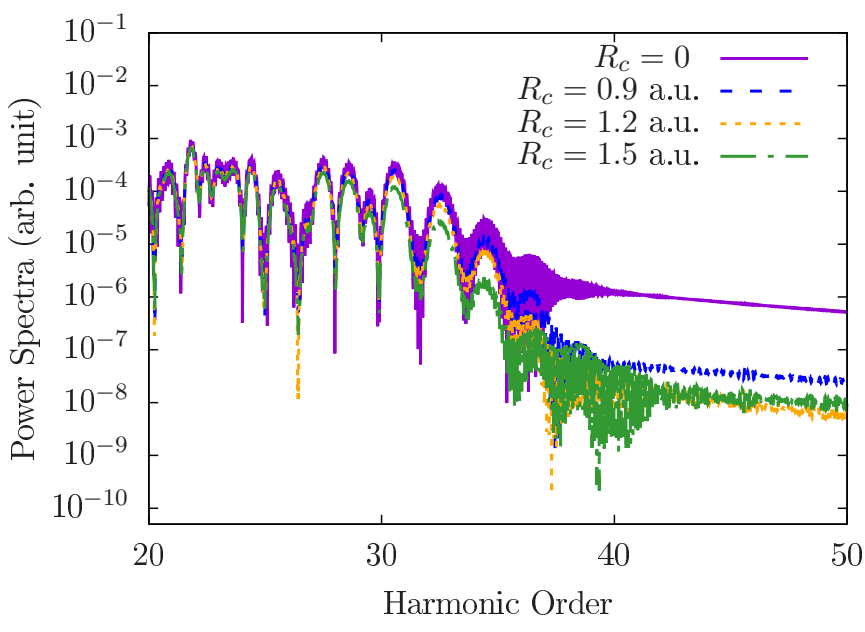

FIG. 8. (Color online) High-order-harmonic generation of $\mathrm{Ar}$ atoms calculated by TDDFT-AE with different $R_{c}$. Laser parameters: $\lambda=800 \mathrm{~nm}, I=2 \times 10^{14} \mathrm{~W} / \mathrm{cm}^{2}, \tau=6 \mathrm{fs}$.

Thus, the dominant contribution to the time-dependent induced dipole is due to the overlap of the initial wave function with the continuum in the region for $r>1.2$ a.u. This explains why the HHG spectra from the AE and NCPP simulations are close to each other since the initial wave functions from $\mathrm{AE}$ and NCPP are close to each other in the region of $r>$ 1.2 a.u. More precisely, the transition dipole is dominated by contributions from $r>1.2$ a.u. The latter point is nicely illustrated by the shape of the pseudo-wave-functions (Fig. 1). While the radial $3 p$ wave functions peak near $r=1.2$ a.u., suggesting significant contributions also from $r<1.2$ a.u., the major contribution to the transition dipole stems from the coupling between the $3 p$ state and the $d$-partial wave. The latter effectively does not penetrate the core region because of the large centrifugal barrier (Fig. 1) and thus shifts the weight in Eq. (16) to larger $r$. Only for large energies of the recolliding electron does core penetration become important.

For delineating the applicability of the TDDFT-PP method it is instructive to consider the transition dipole-matrix element for the radiative recombination, i.e., the last step of the three-step model as a function of the recombination energy. We therefore calculate the transition dipole-matrix elements of $\operatorname{Ar} \epsilon p \rightarrow 3 s$ and $\epsilon s(d) \rightarrow 3 p$ (Fig. 9). The AE and PP transition matrix elements agree with each other at low energies while they start to diverge from each other at higher energies ( $E \geqslant 1.1$ a.u.) corresponding to $n \gtrsim 20$. Such discrepancies are magnified when the transition matrix element features pronounced structures such as in the present case $\operatorname{Ar}(d \rightarrow 3 p)$ a pronounced Cooper minimum near $36 \mathrm{eV}$ [72,73]. Accurately representing the Cooper minimum requires high-level electronic structure calculations. For the $d \rightarrow 3 p$ transition the position of the Cooper minimum is well reproduced by the TDDFT-AE calculation while the TDDFT-PP calculation shifts it to higher energies $(\sim 44 \mathrm{eV})$. The present TDDFT calculation fails to reproduce the Cooper minimum in the $p \rightarrow 3 s$ channels which requires improved treatment of correlation effects, e.g., on the random-phase approximation with exchange level [74]. Our present calculations suggest therefore the approximate agreement between the 


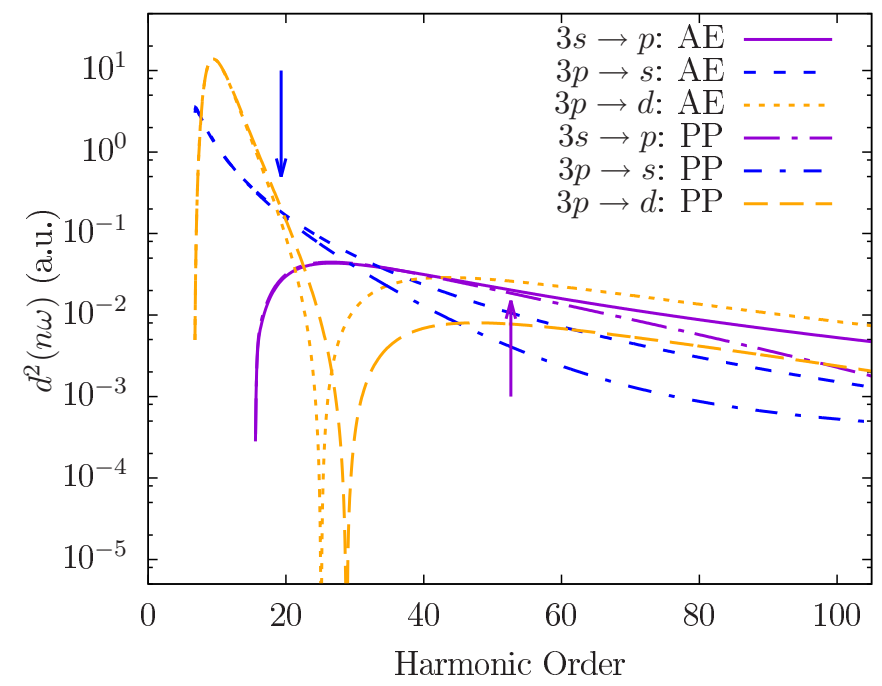

FIG. 9. (Color online) Transition dipole-matrix elements in $\mathrm{Ar}$ atoms calculated with the all-electron potential and norm-conserving pseudopotentials.

transition dipole-matrix elements for radiative recombination in the PP and AE calculations as a useful criterion for the applicability of the pseudopotential method for a given spectral range of $\mathrm{HHG}$.

\section{CONCLUSION}

In summary, we have studied the region of validity of norm-conserving pseudopotentials in laser-matter interactions and found that NCPPs work well for strong-field ionization over a broad range of energies and intensities when comparing TDDFT with all electron and NCPP simulations. For highorder-harmonic generations, NCPP works well for lower order HHGs but may break down for very-high-order HHGs. The criterion for the applicability of NCPPs to HHG can be deduced from the laser-field free transition dipole-matrix elements from AE and NCPP calculations. The present study underscores the applicability of NCPP to laser-matter interactions and lays the foundation for the application of NCPPs to larger system such as molecules, clusters, and solids.

\section{ACKNOWLEDGMENTS}

This research was supported by Grant-in-Aid for Scientific Research C24540421 from the Japan Society for the Promotion of Science and HA-PACS Project for advanced interdisciplinary computational sciences by exa-scale computing technology and by the FWF (Austria), SFB-041 ViCoM, SFB-049 Next Lite, doctoral college W1243, and P21141N16. G.W. thanks the IMPRS-APS for financial support. K.Y. acknowledges support by the Grants-in-Aid for Scientific Research Nos. 23340113 and 25104702 from the Japan Society for the Promotion of Science.
[1] F. Krausz and M. Ivanov, Attosecond physics, Rev. Mod. Phys. 81, 163 (2009).

[2] Th. Fennel, K.-H. Meiwes-Broer, J. Tiggesbäumker, P.-G. Reinhard, P. M. Dinh, and E. Suraud, Laser-driven nonlinear cluster dynamics, Rev. Mod. Phys. 82, 1793 (2010).

[3] A. Di Piazza, C. Müller, K. Z. Hatsagortsyan, and C. H. Keitel, Extremely high-intensity laser interactions with fundamental quantum systems, Rev. Mod. Phys. 84, 1177 (2012).

[4] W. Becker, X.-J. Liu, P. J. Ho, and J. H. Eberly, Theories of photoelectron correlation in laser-driven multiple atomic ionization, Rev. Mod. Phys. 84, 1011 (2012).

[5] P. Hohenberg and W. Kohn, Inhomogeneous electron gas, Phys. Rev. 136, B864 (1964).

[6] W. Kohn and L. J. Sham, Self-consistent equations including exchange and correlation effects, Phys. Rev. 140, A1133 (1965).

[7] W. Kohn, Nobel lecture: Electronic structure of matter-wave functions and density functionals, Rev. Mod. Phys. 71, 1253 (1999).

[8] E. Runge and E. K. U. Gross, Density-Functional Theory for Time-Dependent Systems, Phys. Rev. Lett. 52, 997 (1984).

[9] C. A. Ullrich, U. J. Gossmann, and E. K. U. Gross, TimeDependent Optimized Effective Potential, Phys. Rev. Lett. 74, 872 (1995).

[10] X.-M. Tong and Shih-I Chu, Time-dependent density-functional theory for strong-field multiphoton processes: Application to the study of the role of dynamical electron correlation in multiple high-order-harmonic generation, Phys. Rev. A 57, 452 (1998).
[11] P. Wopperer, P. M. Dinh, P.-G. Reinhard, and E. Suraud, Electrons as probes of dynamics in molecules and clusters: A contribution from time dependent density functional theory, Phys. Rep. 562, 1 (2015).

[12] M. Lein, E. K. U. Gross, and V. Engel, Intense-Field Double Ionization of Helium: Identifying the Mechanism, Phys. Rev. Lett. 85, 4707 (2000).

[13] Xi Chu and Shih-I Chu, Time-dependent density-functional theory for molecular processes in strong fields: Study of multiphoton processes and dynamical response of individual valence electrons of $\mathrm{N}_{2}$ in intense laser fields, Phys. Rev. A 64, 063404 (2001).

[14] D. A. Telnov, K. E. Sosnova, E. Rozenbaum, and ShihI Chu, Exterior complex scaling method in time-dependent density-functional theory: Multiphoton ionization and highorder-harmonic generation of Ar atoms, Phys. Rev. A 87, 053406 (2013).

[15] J. Heslar, D. A. Telnov, and Shih-I Chu, Subcycle dynamics of high-harmonic generation in valence-shell and virtual states of $\mathrm{Ar}$ atoms: A self-interaction-free time-dependent density-functional-theory approach, Phys. Rev. A 91, 023420 (2015)

[16] Xiao-Min Tong and Shih-I Chu, Theoretical study of multiple high-order-harmonic generation by intense ultrashort pulsed laser fields: A new generalized pseudospectral time-dependent method, Chem. Phys. 217, 119 (1997).

[17] D. A. Telnov and Shih-I Chu, $A b$ initio study of highorder-harmonic generation of $\mathrm{H}_{2}{ }^{+}$in intense laser fields: 
Time-dependent non-Hermitian Floquet approach, Phys. Rev. A 71, 013408 (2005).

[18] Y.-J. Jin, X.-M. Tong, and N. Toshima, Enhanced ionization of hydrogen molecular ions in an intense laser field via a multiphoton resonance, Phys. Rev. A 81, 013408 (2010).

[19] M. W. Schmidt, K. K. Baldridge, J. A. Boatz, S. T. Elbert, M. S. Gordon, J. H. Jensen, S. Koseki, N. Matsunaga, K. A. Nguyen, $\mathrm{S}$. Su et al., General atomic and molecular electronic structure system, J. Comput. Chem. 14, 1347 (1993).

[20] S. Mohr, L. E. Ratcliff, P. Boulanger, L. Genovese, D. Caliste, T. Deutsch, and S. Goedecker, Daubechies wavelets for linear scaling density functional theory, J. Chem. Phys. 140, 204110 (2014).

[21] K. Yabana and G. F. Bertsch, Time-dependent local-density approximation in real time, Phys. Rev. B 54, 4484 (1996).

[22] T. Otobe, K. Yabana, and J.-I. Iwata, First-principles calculations for the tunnel ionization rate of atoms and molecules, Phys. Rev. A 69, 053404 (2004).

[23] D. R. Hamann, M. Schlüter, and C. Chiang, Norm-Conserving Pseudopotentials, Phys. Rev. Lett. 43, 1494 (1979).

[24] D. R. Hamann, Generalized norm-conserving pseudopotentials, Phys. Rev. B 40, 2980 (1989).

[25] E. L. Shirley, D. C. Allan, R. M. Martin, and J. D. Joannopoulos, Extended norm-conserving pseudopotentials, Phys. Rev. B 40, 3652 (1989).

[26] C. J. Smallwood, R. E. Larsen, W. J. Glover, and B. J. Schwartz, A computationally efficient exact pseudopotential method. I. Analytic reformulation of the Phillips-Kleinman theory, J. Chem. Phys. 125, 074102 (2006).

[27] N. Troullier and J. L. Martins, Efficient pseudopotentials for plane-wave calculations, Phys. Rev. B 43, 1993 (1991).

[28] R. Nagano, K. Yabana, T. Tazawa, and Y. Abe, Application of the time-dependent local density approximation to collision between a highly charged ion and an atom, J. Phys. B 32, L65 (1999).

[29] K. Nobusada and K. Yabana, High-order harmonic generation from silver clusters: Laser-frequency dependence and the screening effect of $d$ electrons, Phys. Rev. A 70, 043411 (2004).

[30] G. Wachter, C. Lemell, J. Burgdörfer, Shunsuke A. Sato, X.-M. Tong, and K. Yabana, Ab Initio Simulation of Electrical Currents Induced by Ultrafast Laser Excitation of Dielectric Materials, Phys. Rev. Lett. 113, 087401 (2014).

[31] K. Yabana and G. F. Bertsch, Oscillator strengths with pseudopotentials, Phys. Rev. A 58, 2604 (1998).

[32] P. A. M. Dirac, Note on exchange phenomena in the Thomas atom, Math. Proc. Cambridge 26, 376 (1930).

[33] S. H. Vosko, L. Wilk, and M. Nusair, Accurate spin-dependent electron liquid correlation energies for local spin density calculations: A critical analysis, Can. J. Phys. 58, 1200 (1980).

[34] F. Tran and P. Blaha, Accurate Band Gaps of Semiconductors and Insulators with a Semilocal Exchange-Correlation Potential, Phys. Rev. Lett. 102, 226401 (2009).

[35] V. U. Nazarov and G. Vignale, Optics of Semiconductors from Meta-Generalized-Gradient-Approximation-Based TimeDependent Density-Functional Theory, Phys. Rev. Lett. 107, 216402 (2011)

[36] X.-M. Tong and Shih-I Chu, Density-functional theory with optimized effective potential and self-interaction correction for ground states and autoionizing resonances, Phys. Rev. A 55, 3406 (1997).
[37] G. Duchateau, E. Cormier, H. Bachau, and R. Gayet, CoulombVolkov approach of atom ionization by intense and ultrashort laser pulses, Phys. Rev. A 63, 053411 (2001).

[38] M. V. Volkov and V. N. Ostrovsky, Exact results for survival probability in the multistate landau-zener model, J. Phys. B 37, 4069 (2004).

[39] D. G. Arbó, J. E. Miraglia, M. S. Gravielle, K. Schiessl, E. Persson, and J. Burgdörfer, Coulomb-Volkov approximation for near-threshold ionization by short laser pulses, Phys. Rev. A 77, 013401 (2008).

[40] X. M. Tong, K. Hino, and N. Toshima, Phase-dependent atomic ionization in few-cycle intense laser fields, Phys. Rev. A 74, 031405 (2006).

[41] U. De Giovannini, D. Varsano, M. A. L. Marques, H. Appel, E. K. U. Gross, and A. Rubio, Ab initio angleand energy-resolved photoelectron spectroscopy with timedependent density-functional theory, Phys. Rev. A 85, 062515 (2012).

[42] X.-M. Tong, P. Ranitovic, D. D. Hickstein, M. M. Murnane, H. C. Kapteyn, and N. Toshima, Enhanced multiple-scattering and intra-half-cycle interferences in the photoelectron angular distributions of atoms ionized in midinfrared laser fields, Phys. Rev. A 88, 013410 (2013).

[43] R. Stowasser and R. Hoffmann, What do the Kohn-Sham orbitals and eigenvalues mean? J. Am. Chem. Soc. 121, 3414 (1999).

[44] V. P. Krainov, Ionization rates and energy and angular distributions at the barrier-suppression ionization of complex atoms and atomic ions, J. Opt. Soc. Am. B 14, 425 (1997).

[45] X. M. Tong, Z. X. Zhao, and C. D. Lin, Theory of molecular tunneling ionization, Phys. Rev. A 66, 033402 (2002).

[46] X. M. Tong and C. D. Lin, Empirical formula for static field ionization rates of atoms and molecules by lasers in the barriersuppression regime, J. Phys. B 38, 2593 (2005).

[47] J. J. Carrera, Shih-I Chu, and X. M. Tong, Very-high-orderharmonic generation from $\mathrm{Ar}$ atoms and $\mathrm{Ar}^{+}$ions in superintense pulsed laser fields: An $a b$ initio self-interaction-free time-dependent density-functional approach, Phys. Rev. A 71, 063813 (2005).

[48] N. Sarukura, K. Hata, T. Adachi, R. Nodomi, M. Watanabe, and $\mathrm{S}$. Watanabe, Coherent soft-x-ray generation by the harmonics of an ultrahigh-power KrF laser, Phys. Rev. A 43, 1669 (1991).

[49] E. A. Gibson, A. Paul, N. Wagner, R. Tobey, S. Backus, I. P. Christov, M. M. Murnane, and H. C. Kapteyn, High-Order Harmonic Generation up to $250 \mathrm{eV}$ from Highly Ionized Argon, Phys. Rev. Lett. 92, 033001 (2004).

[50] D. N. Fittinghoff, P. R. Bolton, B. Chang, and K. C. Kulander, Observation of Nonsequential Double Ionization of Helium with Optical Tunneling, Phys. Rev. Lett. 69, 2642 (1992).

[51] K. Kondo, A. Sagisaka, T. Tamida, Y. Nabekawa, and S. Watanabe, Wavelength dependence of nonsequential double ionization in He, Phys. Rev. A 48, R2531 (1993).

[52] B. Walker, B. Sheehy, L. F. DiMauro, P. Agostini, K. J. Schafer, and K. C. Kulander, Precision Measurement of Strong Field Double Ionization of Helium, Phys. Rev. Lett. 73, 1227 (1994).

[53] A. Becker and F. H. M. Faisal, Mechanism of laser-induced double ionization of helium, J. Phys. B 29, L197 (1996).

[54] B. Feuerstein, R. Moshammer, and J. Ullrich, Nonsequential multiple ionization in intense laser pulses: Interpretation of 
ion momentum distributions within the classical 'rescattering' model, J. Phys. B 33, L823 (2000).

[55] T. Ishikawa, X. M. Tong, and N. Toshima, Double ionization of $\mathrm{He}$ in an intense laser field via a rescattering process, Phys. Rev. A 82, 033411 (2010).

[56] G. G. Paulus, W. Becker, W. Nicklich, and H. Walther, Rescattering effects in above-threshold ionization: A classical model, J. Phys. B 27, L703 (1994).

[57] N. B. Delone and V. P. Krainov, Tunneling and barriersuppression ionization of atoms and ions in a laser radiation field, Phys. Usp. 41, 469 (1998).

[58] P. Antoine, A. L'Huillier, and M. Lewenstein, Attosecond Pulse Trains using High-Order Harmonics, Phys. Rev. Lett. 77, 1234 (1996).

[59] P. Corkum, Laser physics: Attosecond pulses at last, Nature 403, 845 (2000).

[60] Z. Chang, Single attosecond pulse and xuv supercontinuum in the high-order-harmonic plateau, Phys. Rev. A 70, 043802 (2004).

[61] J. Itatani, F. Quéré, G. L. Yudin, M. Yu. Ivanov, F. Krausz, and P. B. Corkum, Attosecond Streak Camera, Phys. Rev. Lett. 88, 173903 (2002).

[62] G. Sansone, F. Kelkensberg, J. F. Perez-Torres, F. Morales, M. F. Kling, W. Siu, O. Ghafur, P. Johnsson, M. Swoboda, E. Benedetti, F. Ferrari, F. Lepine, J. L. Sanz-Vicario, S. Zherebtsov, I. Znakovskaya, A. L'Huillier, M. Yu. Ivanov, M. Nisoli, F. Martin, and M. J. J. Vrakking, Electron localization following attosecond molecular photoionization, Nature $\mathbf{4 6 5}$, 763 (2010)

[63] P. Ranitovic, C. W. Hogle, P. Rivière, A. Palacios, X.-M. Tong, N. Toshima, A. González-Castrillo, L. Martin, F. Martín, M. M. Murnane, and H. Kapteyn, Attosecond vacuum UV coherent control of molecular dynamics, Proc. Natl. Acad. Sci. USA 111, 912 (2014).
[64] X. Guan, X.-M. Tong, and Shih-I Chu, Effect of electron correlation on high-order-harmonic generation of helium atoms in intense laser fields: Time-dependent generalized pseudospectral approach in hyperspherical coordinates, Phys. Rev. A 73, 023403 (2006).

[65] J. C. Baggesen and L. B. Madsen, On the dipole, velocity and acceleration forms in high-order-harmonic generation from a single atom or molecule, J. Phys. B 44, 115601 (2011).

[66] A. Rundquist, C. G. Durfee, Z. Chang, C. Herne, S. Backus, M. M. Murnane, and H. C. Kapteyn, Phase-matched generation of coherent soft X-rays, Science 280, 1412 (1998).

[67] J. L. Krause, K. J. Schafer, and K. C. Kulander, High-Order Harmonic Generation from Atoms and Ions in the High Intensity Regime, Phys. Rev. Lett. 68, 3535 (1992).

[68] P. B. Corkum, Plasma Perspective on Strong Field Multiphoton Ionization, Phys. Rev. Lett. 71, 1994 (1993).

[69] Anh-Thu Le, R. R. Lucchese, S. Tonzani, T. Morishita, and C. D. Lin, Quantitative rescattering theory for high-orderharmonic generation from molecules, Phys. Rev. A 80, 013401 (2009).

[70] A. Zangwill and P. Soven, Density-functional approach to localfield effects in finite systems: Photoabsorption in the rare gases, Phys. Rev. A 21, 1561 (1980).

[71] M. Lewenstein, Ph. Balcou, M. Yu. Ivanov, A. L'Huillier, and P. B. Corkum, Theory of high-harmonic generation by lowfrequency laser fields, Phys. Rev. A 49, 2117 (1994).

[72] J. W. Cooper, Photoionization from outer atomic subshells. A model study, Phys. Rev. 128, 681 (1962).

[73] B. Möbus, B. Magel, K.-H. Schartner, B. Langer, U. Becker, M. Wildberger, and H. Schmoranzer, Measurements of absolute Ar $3 s$ photoionization cross sections, Phys. Rev. A 47, 3888 (1993).

[74] A. S. Kheifets, Time delay in valence-shell photoionization of noble-gas atoms, Phys. Rev. A 87, 063404 (2013). 